\title{
Krebs und Ernährung
}

\section{Ist Kalzium tatsächlich karzinogen für die Prostata?}

\begin{abstract}
— Die Hypothese, dass mit der Aufnahme von Kalzium das Risiko für ein Prostatakarzinom zunimmt, wird durch eine Fall-KontrollStudie aus den USA nicht bestätigt [Williams CD et al. Prev Chronic Dis 2012 doi: 10.5888/ pcd9.110125]. In der Fall-Kontroll-Studie wurden drei Personengruppen verglichen: 108 Patienten mit bioptisch bestätigtem Prostatakarzinom, 161 Patienten mit entsprechendem klinischem Verdacht, aber negativem Biopsieergebnis und 237 gesunde Kontrollpersonen. Alle Teilnehmer hatten ausführliche Fragebögen zu ihrer Ernährung und zu bekannten Risikofaktoren des Prostatakarzinoms ausgefüllt.

Die Kalziumzufuhr insgesamt, aus Mahlzeiten, Kalziumtabletten und Multivitamin-
\end{abstract}

präparaten, lag im Mittel bei etwa $800 \mathrm{mg}$ pro Tag - und damit etwa 200 mg niedriger als üblicherweise für Erwachsene empfohlen. Wie der Vergleich mit jeder der beiden Kontrollgruppen ergab, war eine kalziumreichere Ernährung mit einer geringeren Prostatakrebsrate assoziiert - allerdings nur in Bezug auf hochgradige Prostatakarzinome (Gleason-Score $>$ 7).

Zudem lag die ernährungsabhängige Kalziumaufnahme auch in der höchsten Terzile nur bei gut $1.000 \mathrm{mg}$ pro Tag. Die Studie lässt daher keine Aussage darüber zu, ob sehr große Kalziummengen nicht doch - wie in früheren Studien beobachtet - zur Progression von Prostatakrebs beitragen können.

Dr. Beate Schumacher

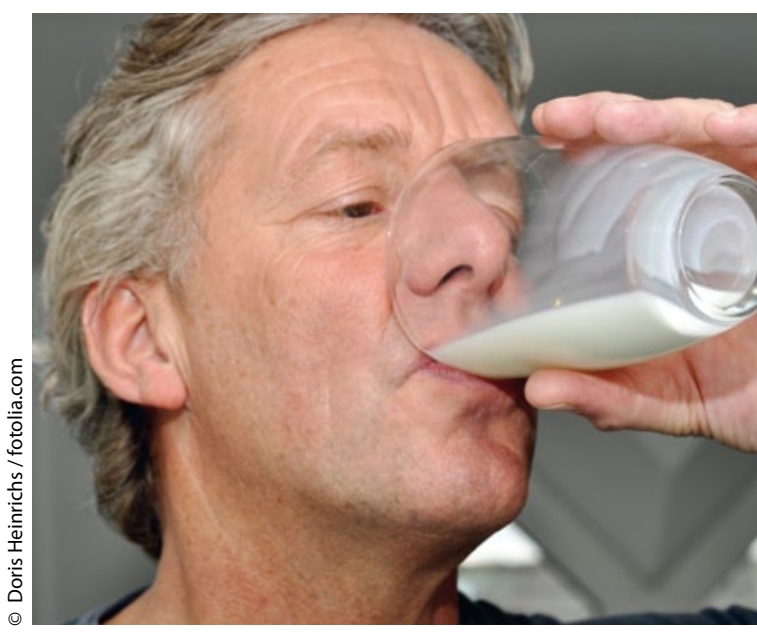

Wie viel Kalziumaufnahme nützt der Prostata?

\section{Neue EAU-Leitlinien zur Vasektomie}

\section{Sieben Punkte für das Aufklärungsgespräch}

— Die Vasektomie ist männlicherseits das verlässlichste Mittel der Empfängnisverhütung - aber ihr Einsatz muss wohl überlegt sein. Die Europäische Gesellschaft für Urologie (EAU) hat nun Leitlinien vorgelegt [Dohle GR et al. Eur Urol 2011; doi:10.1016/ j.eurouro.2011.10.00].

Gemäß den neuen EAU-Leitlinien sollte der Arzt

1. sichergehen, dass der Patient freiwillig auf die Fortpflanzung verzichtet;

2.über den Gesundheitszustand des Patienten informiert sein;

3. dem Patienten die eingesetzte Vasektomietechnik einschließlich Vor- und Nachteilen erläutern;

4. berücksichtigen, dass jüngeres Alter ( $<30$ Jahre) und aktuelle Partnerlosigkeit relative Kontraindikationen darstellen;

5. sowohl Alternativen zu Vasektomie als auch mögliche Komplikationen, Erfolgsraten und die Möglichkeit eines Fehlschlags diskutieren;

6. darauf hinweisen, dass bis zum Nachweis der Sterilität Verhütungsmaßnahmen erforderlich sind und drei Monate nach dem Eingriff eine Samenanalyse vorgenommen werden muss;

7. den Patienten (schriftlich) über den Ablauf der Samenanalyse einschließlich Ergebnismitteilung informieren.

Der Arzt muss auch deutlich machen, dass es keine 100\%ige Garantie auf permanente Sterilität geben kann. Für den Eingriff selbst werden folgende Maßnahmen empfohlen: Exzision jeweils eines Ductus-deferens-Abschnitts und Ligatur mit Nähten oder Clips, Interposition von Gewebe, um einer Rekanalisation vorzubeugen, Kauterisieren des Samenleiterlumens. Die eingesetzte Technik einschließlich Sicherheitsmaßnahmen sind in einem Standard-OP-Bericht zu dokumentieren. Wundkontrollen sind in der Regel nicht erforderlich, der Patient sollte aber wissen, wen er bei Problemen kontaktieren kann.

Grünes Licht für ungeschützten Sex kann der Arzt geben, wenn nach drei Monaten entweder keine oder weniger als 100.000 nicht mobile Spermien im Ejakulat nachweisbar sind.

Dr. Elke Oberhofer
Titelbild des Jahres 2011

And the winner is ...

— Sie haben gewählt! $42 \%$ der Stimmen fielen bei der Wahl zum Titelbild des Jahres 2011 auf Ausgabe 10 (Schwerpunkt Urolithiasis).

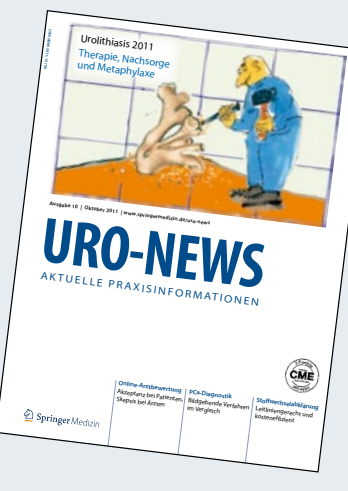

Wir gratulieren den Gewinnern: Das Buch „Urogenitale Bildgebung" geht an Roman Karig aus Düsseldorf, das Buch „Batman und andere himmlische Kreaturen" an Stefan Kowalik aus Apolda und der WLAN-Router Linksys E4200 von Cisco an Heiko Otto aus Dieburg.

Herzlichen Glückwunsch, Ihre URO-NEWS-Redaktion

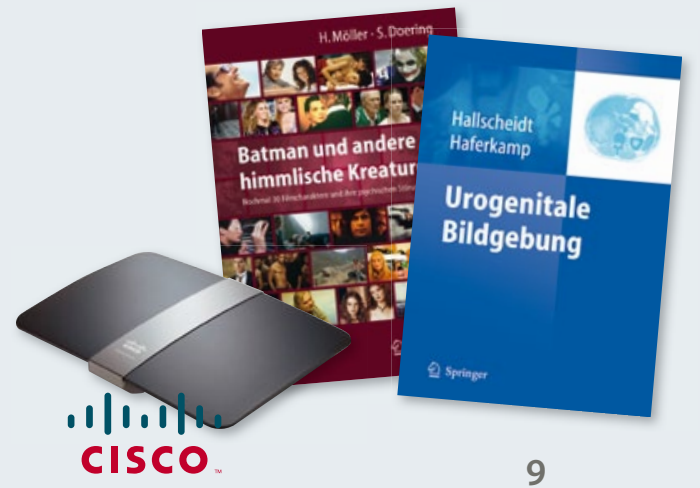

